\title{
Problemas de sono em idade pediátrica
}

Manuel Salavessa,* Paula Vilariça**

\section{RESUMO}

A importância e impacto do sono na saúde em geral têm merecido, nos últimos tempos, uma maior atenção, sendo os problemas do sono fonte comum de preocupação por parte dos pais. O sono desempenha um papel fundamental sob ponto de vista biológico, mas também emocional e social. Os problemas de sono podem ter um impacto negativo significativo na criança e família. Propomo-nos com este artigo tratar informação básica acerca de problemas de sono comuns em idade pediátrica, para ser usado pelo clínico quando confrontado com as preocupações dos pais. Apresentamos neste artigo aspectos fundamentais da arquitectura normal e do desenvolvimento dos padrões de sono, seguindo-se uma exposição acerca dos problemas de sono clinicamente mais comuns em crianças e adolescentes.

Palavras-chave: Sono; Perturbação do Sono; Criança; Adolescente.

\section{INTRODUÇÃO}

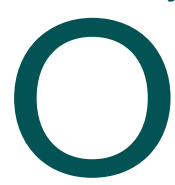

sono é uma área do funcionamento da criança que ocupa grande parte da sua vida e se apresenta como um desafio para pais e crianças. O sono é essencial para o normal crescimento e desenvolvimento, quer nos aspectos físicos, quer emocionais da criança. ${ }^{1} \mathrm{O}$ sono desempenha, para lá da sua função biológica, um papel importante na modulação da relação precoce entre os pais e o seu bebé, sendo um passo fundamental na aprendizagem da sua separação. O sono favorece, em condições normais, no bebé, o desenvolvimento de processos de auto-regulação fundamentais para o seu desenvolvimento emocional e interacções sociais futuras. ${ }^{2}$ Apesar do momento do sono ser, em regra, um momento recheado de sensações prazerosas, muitas das vezes pode transformar-se num momento particularmente difícil e angustiante, com possível deterioração na relação precoce entre pais e filhos, com eventual compromisso do desenvolvimento.

Os problemas do sono são comuns (20-30\%) na infância, ${ }^{1}$ na idade escolar e na adolescência, sendo fonte comum de preocupação por parte dos pais. ${ }^{4}$ Nas suas formas mais graves, e independentemente da idade,

* Médico Interno de Psiquiatria da Infância e Adolescência, Hospital D. Estefânia, Centro Hospitalar Lisboa Central, EPE

**Assistente Hospitalar de Psiquiatria da Infância e Adolescência, Unidade da Primeira Infância, Hospital D. Estefânia, Centro Hospitalar Lisboa Central, EPE causam impacto significativo no dia-a-dia das crianças e famílias, quer do ponto de vista social, quer comportamental, emocional e académico. ${ }^{1}$

Apesar de comuns na prática clínica, os problemas do sono são frequentemente desvalorizados ou erroneamente diagnosticados. ${ }^{5}$ Tal deve-se a uma escassa formação e informação por parte dos clínicos, ${ }^{1}$ traduzindo-se muitas vezes num uso abusivo de fármacos em idades pediátricas. ${ }^{4}$ Na presença de problemas do sono, torna-se importante reconhecer se a situação é transitória e auto-limitada, ligada a uma fase de desenvolvimento, se é uma perturbação de sono per si ou se existe uma doença orgânica ou psiquiátrica subjacente ao problema.

Deste modo este artigo visa contribuir para uma melhor compreensão desta problemática quer no respeitante ao diagnóstico das perturbações de sono mais comuns e quer no respeitante à atitude terapêutica destas alterações.

Para tal iremos abordar brevemente a fisiologia do sono, debruçando-nos sobre a sua arquitectura e desenvolvimento dos padrões de sono, seguindo-se uma exposição acerca dos problemas de sono clinicamente mais comuns em crianças e adolescentes.

\section{FISIOLOGIA DO SONO}

O sono é um estado comportamental reversível caracterizado por uma diminuição de resposta e interacção 
com o ambiente. ${ }^{1,2} \mathrm{O}$ sistema neurológico que comanda as alterações cíclicas sono-vigília estende-se desde a medula, passando pelo tronco cerebral e hipotálamo até núcleos centrais superiores. ${ }^{7} \mathrm{~A}$ função fisiológica do sono ainda é desconhecida. As duas teorias mais populares defendem que o sono é essencial para a restituição das funções cerebrais ou reabastecimento do corpo, visto que o sono, e a sua imobilidade imposta, se traduzem num corte dos gastos energéticos. ${ }^{2}$ No entanto o sono não se traduz por um "descanso» do corpo, sendo um período de actividade cerebral intensa, envolvendo funções corticais superiores. Mais recentemente foram identificadas funções importantes ao nível da memória, bem como da optimização das funções imunológicas. ${ }^{1}$

O padrão de actividade eléctrica do Sistema Nervoso Central (SNS), tónus muscular e movimento ocular permite dividir o sono em dois tipos, (1) sono REM ( $\mathrm{ra}$ pid eye movement) e (2) sono NREM (non-REM), com diferentes estádios dentro do sono NREM.

O sono NREM divide-se em 4 estádios: 1 a 4 . Os estádios 1 e 2 correspondem ao sono leve. Os estádios 3 e 4 são normalmente combinados, apresentam um registo eléctrico típico com ondas lentas, apelidadas de ondas delta, e são denominados sono de ondas lentas. Este é o sono mais profundo nos seres humanos e ocorre predominantemente na primeira metade da noite. Durante este estádio as crianças dificilmente são acordadas, e se isto acontece, parecem confusas e desorientadas. É durante este estádio de sono profundo que podem ocorrer as parassónias como o sonambulismo, sonilóquios e terrores nocturnos.

O sono REM partilha características de sono profundo com características de sono leve. Ocorre em ciclos de cerca de 90 minutos cada sendo mais comum na segunda metade da noite. No sono REM há um relaxamento do tónus muscular, excepto nos recém-nascidos, em que se observam pequenos movimentos das extremidades ou da face. $\mathrm{O}$ sono REM corresponde à fase de sonhos, caracterizando-se por uma intensa actividade cortical.

O sono não é um estado uniforme, existindo uma alternância entre ciclos de sono REM e NREM, interrompidos por breves momentos de despertares, a maioria dos quais passa despercebido a observadores e ao próprio e que são caracterizados por registos electroence- falográficos perto do estado de vigília. ${ }^{2}$ A maioria das crianças desenvolve processos auto-regulatórios que lhes permite adormecer novamente após estes períodos de despertar. ${ }^{8}$ Cada ciclo de sono demora cerca de 50-60 minutos no recém-nascido, gradualmente aumentando para 90 minutos na adolescência. ${ }^{6} \mathrm{Na}$ criança, o sono inicia-se na fase NREM, com predomínio dos estádios 3 e 4 na primeira parte da noite e com períodos breves de sono REM. Com o avanço da noite, os estádios 1 e 2 de NREM, bem como o sono REM, vão ganhando maior predominância em cada ciclo, até ao despertar matinal, normalmente de sono REM ou dos estádios de sono leve (1 e 2) do NREM.

A proporção entre sono REM E NREM vai mudando com o crescimento, acompanhando a maturação do SNC. Assim, o recém-nascido frequentemente inicia a noite em sono REM, sendo que a proporção entre sono REM e NREM vai diminuindo com o crescimento. ${ }^{3}$

Em relação ao desenvolvimento do ciclo sono vigília, acredita-se que se origine ainda na vida fetal. ${ }^{2} \mathrm{O}$ feto parece nunca estar, de facto, acordado, alternando entre um sono calmo e sono activo, precursores do sono NREM e REM. ${ }^{2}$ Com o nascimento e o rápido desenvolvimento que se segue, uma das funções primordiais é a sincronização fisiológica do bebé com as relações sociais primárias. Numa primeira fase, o recém-nascido alterna entre sono e vigília a cada $3-4 \mathrm{~h}$, com despertares geralmente relacionados com a fome. ${ }^{6}$ Os períodos de vigília vão aumentando gradualmente durante o dia. Por volta da $5^{\text {a }}$ semana de vida estabelece-se um ciclo de vigília diurna na maioria dos bebés. ${ }^{5,6}$ Estudos realizados, em que os pais preenchem questionários sobre o sono dos filhos, revelam que aos 3 meses cerca de $70 \%$ dos bebés são capazes de dormir durante a noite, o que corresponde a um período de sono contínuo de cerca de 5 horas. ${ }^{1,5} \mathrm{O}$ estabelecimento deste ciclo depende da organização e maturação dos processos superiores do SNC, pelo que recém-nascidos prematuros, ou que tenham sofrido lesões no período peri-natal, como anoxia, desenvolvem esta capacidade mais tardiamente. Este relógio biológico, que regula o ciclo, sincroniza-se com outros sinais internos (fome e saciedade), bem como com os períodos de interacção social e com outros sinais do ambiente, como o ciclo de luz-escuridão e a temperatura ambiente. ${ }^{4}$ Geralmente o padrão de sono estável só emerge a partir dos 12 meses. ${ }^{1}$ 
As necessidades diárias de sono decrescem lentamente nestes primeiros meses (16,3 horas diárias ao nascer para 14,8 horas diárias aos 16 meses), representando sobretudo o decréscimo do sono diurno, com aumento da vigília diurna e diminuição do número de sestas. ${ }^{2,5}$ À noite e aos 16 meses o sono contínuo aumenta, em média, das 4 para as 8,5 horas. ${ }^{2}$ Por volta dos 2 -3 anos a necessidade de sono reduz-se para 12 horas diárias, com decréscimo para as 9 horas diárias entre os 9 e os 12 anos. ${ }^{2,5}$ Durante a adolescência parece que as necessidades de sono aumentam ligeiramente, para decair para as 8 horas diárias no jovem adulto. ${ }^{2,5}$ É de salientar que a maioria dos estudos realizados sobre sono infantil se baseia nas informações fornecidas pelos prestadores de cuidados das crianças (geralmente os pais), através de entrevistas ou preenchimento de questionários, sendo raros os que usam medidas directas (polissonografia) para medição das horas de sono em populações normais. Estes últimos, na maioria, são realizados com populações pequenas e têm resultados pouco generalizáveis. ${ }^{1}$ A questão das horas normais de sono em bebés e crianças permanece incompletamente resolvida, dando lugar a uma multiplicidade de interpretações pessoais dos técnicos que lidam estas problemáticas. O mais consensual é que as necessidades de sono são variáveis de indivíduo para indivíduo, dependentes das suas idiossincrasias, constituindo os números apresentados médias que pretendem servir de orientação geral. ${ }^{1}$

\section{PERTURBAÇÕES DO SONO}

De acordo com a $4^{\mathrm{a}}$ edição revista da Diagnostic and Statistical Manual of Mental Disorders, de 2002 (DSM IV-TR), classificação diagnóstica da Associação Americana de Psiquiatria, as perturbações do sono dividem-se em dissónias (caracterizadas por anomalias da quantidade, qualidade ou horário do sono), parassónias (eventos comportamentais ou fisiológicos anormais durante o horário de sono), perturbações do sono relacionadas com perturbações psiquiátricas e perturbações do sono associadas a doença física. Esta classificação diagnóstica, apesar da sua utilidade, foi elaborada centrando-se nas dificuldades de sono nos adultos e tem pouca sensibilidade às especificidades do desenvolvimento, importantes nas crianças e adolescentes. ${ }^{4}$ Clinicamente, crianças e adolescentes com problemas de sono apresentam sintomas tipicamente de uma destas 4 áreas ${ }^{2}(1)$ insónia (dificuldades no adormecimento e/ou despertares nocturnos) (2) sonolência diurna excessiva (3) eventos comportamentais ou fisiológicos anormais durante o horário de sono (parassónias) (4) problemas do sono relacionados com perturbações psiquiátricas ou médicas.

$\mathrm{Na}$ abordagem de uma criança ou adolescente com perturbação do sono, a história clínica reveste-se de importância fundamental. Questões centrais como saber se a criança está a ter dificuldades em adormecer ou manter-se a dormir, ocorrência de episódios comportamentais durante o sono ou existência de sonolência diurna devem ser colocadas à partida aos principais prestadores de cuidados. Se estas dificuldades ocorrerem, deverá ser colhida uma história do sono detalhada, bem como do ciclo sono-vigília, devendo ser abordadas questões fundamentais como sestas diurnas, horário das refeições nocturnas e alimentação, actividade após a última refeição, postura da criança e da família perante a hora de deitar, qualidade do sono, despertares, atitude perante os despertares. É de importância fundamental a realização de um diário do sono durante 2 semanas. ${ }^{1,2} \mathrm{O}$ clínico deverá manter-se recordado que, perante a análise de um caso de dificuldades de sono, terá de pensar na eventualidade de estar perante uma perturbação orgânica ou psiquiátrica cuja apresentação se faz através de problemas no sono. Para tal é necessária uma avaliação cuidadosa do estado físico, emocional e de desenvolvimento da criança.

Apenas num reduzido número de casos são necessários estudos fisiológicos de sono, polissonografias, parecendo ser mais úteis em situações de sonolência diurna excessiva, parassónias complicadas e suspeita de apneia de sono. ${ }^{2}$ A polissonografia inclui electroencefalograma, bem como gravações do tónus e do movimento ocular, permitindo o reconhecimento das diferentes fases do sono.

\section{INSÓNIA}

Dificuldades no adormecimento e despertares nocturnos (características chave da insónia) são queixas comuns dos pais de bebés e crianças mais novas, podendo considerar-se tratar-se de uma situação quase normal, que vai diminuindo de frequência e intensidade ao longo do tempo, até aos 5 anos em crianças nas quais 
o desenvolvimento e o funcionamento global se mantêm preservados. ${ }^{2,8,9}$ Como já referimos, o padrão estável de sono só se define completamente aos 12 meses pelo que antes dessa idade não deverá ser colocado o diagnóstico de Perturbação do Comportamento do Sono (DC 0-3:R, Zero to Three, 2005). Apesar desta limitação do diagnóstico é importante reconhecer que existem bebés nos quais o sono é excessivamente desorganizado e fragmentado, que pode acarretar consequências graves em termos de desenvolvimento e que requerem atenção clínica. Estima-se que entre 3 a 14\% de crianças tenham este tipo de problemas. ${ }^{4}$

O processo de se manter sozinho na sua cama, a aquisição da capacidade de se auto-confortar e adormecer é uma tarefa de aprendizagem para as crianças, que deve ser apoiada pelos prestadores de cuidados ${ }^{10,11}$ mas que depende fundamentalmente de características temperamentais e de reactividade da crianç ${ }^{2,4,8}$. Exige a organização de uma área transicional satisfatória (processo de separação dos pais), depende do temperamento e capacidade de auto-regulação por parte da criança mas, como processo de aprendizagem que é, também depende das condições exteriores e das atitudes parentais. Quando estas competências não são adquiridas, as manifestações clínicas podem ser variadas, desde oposição ao deitar a rituais intermináveis com o objectivo de adiar a hora de deitar, até a dificuldades de adormecimento ou despertares nocturnos frequentes., ${ }^{2,8}$

Assim, na avaliação de perturbações do comportamento do sono/insónia em crianças pequenas devem ser tomadas em conta a existência de factores específicos da criança tais como dificuldades biológicas de auto-regulação ou integração sensorial, como pode ser o caso de crianças hipo ou hipersensíveis à estimulação sensorial, com impacto sobre a sua homeostasia global e com manifestações nas áreas funcionais, como é o sono.

Outros factores importantes a serem avaliados são os problemas psicossociais e do ambiente da criança. ${ }^{4} \mathrm{Em}$ casos em que se verifiquem problemas desta natureza, o tratamento é direccionado para intervenções psicossociais e suporte aos pais, tendo como foco de atenção clínica a relação entre os pais e a criança., ${ }^{2,45}$ Alguns princípios devem ser tidos em conta, o principal será uma atitude de escuta empática em relação às dificul- dades dos pais e nunca uma atitude de culpabilização, cujo efeito poderá ser contraproducente. Por vezes a redução de ansiedade dos pais, através da conversa com alguém que os escuta e compreende, poderá ser o passo mobilizador em torno de um objectivo comum, bem como a criação de uma aliança terapêutica. Geralmente os pais que pedem auxílio para tratamento de uma perturbação de comportamento do sono necessitam de estratégias práticas para lidar com a situação. Muitas vezes a intervenção psicopedagógica com as famílias é suficiente para melhorar significativamente o problema. ${ }^{4}$ Os princípios fundamentais que terão de ser comunicados aos pais são a importância da consistência dos seus comportamentos e da existência de uma rotina, que englobe a regularização dos horários do sono e de outras rotinas diárias. ${ }^{1,10,11} \mathrm{O}$ incentivar a criança desde cedo a adormecer sozinha e em quarto próprio aplica-se à maioria das crianças mas há casos excepcionais em bebés, especialmente os que estão a ser amamentados, que precisam do contacto próximo com a mãe, para se auto-regularem e organizarem o sono. Nestas situações o co-sleeping pode ser uma forma de apoiar a criança neste processo. O co-sleeping é desaconselhado em famílias de pais com problemas de tabagismo, alcoolismo e toxicodependência. O aconselhamento aos pais passa também pela proposta de organização do momento de adormecer para que seja tranquilizante (criar um ambiente tranquilo, temperatura adequada e evitar momentos de excitabilidade / hiperestimulação no momento de deitar) sem criar rituais de adormecer demasiado complicados ou rígidos, que podem tornar-se angustiantes para a criança e para a família. Incentivar o estabelecimento de limites é de extrema importância, de modo a que não se crie um desequilíbrio familiar em torno do problema do sono da criança, que confere à criança, por um lado, uma falsa ideia de omnipotência, mas, por outro, uma sensação de desamparo, pela falha dos pais em conseguirem auto-controlar-se e servirem de verdadeiro motor contentor e ansiolítico para a criança. Para ajudar a criança a encontrar as suas formas de apaziguamento pode ser encorajado o uso de objectos transicionais visto que se trata de objectos investidos de afecto pela criança e que a apoiam no processo de separação dos pais quando passa da vigília para o sono. Todas as estratégias propostas devem ser discutidas com os pais e avaliada a sua 
eficácia, convindo recordar que nem todas são terapêuticas para uma determinada criança ou família. ${ }^{4}$

Em crianças de idade escolar o problema pode manter-se ou aparecer de novo. A manifestação mais comum é a resistência em ir dormir, com recusa ou adiamentos sucessivos da hora de deitar. O problema pode ser perpetuado quando os pais demonstram dificuldades em apoiar a transição da vigília para o sono, que deverá ser feita através de regras consistentes acerca da hora de dormir, e do estabelecimento de limites de uma forma clara, assertiva e não punitiva. ${ }^{2}$ Nestas idades, as crianças poderão desenvolver ainda insónia relacionada com medos e fobias específicas. Torna-se importante nestas situações perceber quais as fontes destes, como primeiro passo no tratamento.

A medicação só tem indicação em situações específicas, não devendo ser prescrita como rotina ${ }^{4} \mathrm{O}$ S fármacos deverão ser usados por breves períodos, como coadjuvantes de uma intervenção em curso, nunca de forma isolada. Os agentes farmacológicos mais usados na prática clínica são a melatonina, hidroxizina, o hidrato de cloral e o clonazepam. ${ }^{4}$

Se as medidas gerais não forem suficientes e a situação provocar uma situação com impacto grave na criança e na família, o caso deverá ser referenciado a consulta de pedopsiquiatria.

Nos adolescentes, pode aparecer, associada aos problemas de sono, uma dificuldade fisiológica relacionada com a hora do deitar, denominada de delayed sleep phase syndrome (DSPS). Nesta situação há uma alteração fisiológica do ritmo circadiano, em que há um atraso na hora de deitar, em norma algumas horas após a meia-noite, desfasada da norma social. Como adormecem tarde, existe uma grande dificuldade em acordar de manhã e cumprir com a obrigação dos horários escolares. Normalmente a única alteração na DSPS centra-se na hora tardia do deitar, não havendo alterações qualitativas no sono. ${ }^{2}$ Apesar de ocorrer tipicamente nos adolescentes, associada às alterações biológicas pubertais e a mudanças de estilo de vida, a DSPS pode ocorrer em qualquer idade. ${ }^{2}$ Esta situação pode ser manejada antecipando primeiro a hora de acordar e posteriormente a hora de dormir, até à hora desejada. Pela mesma ordem poderá adiantar-se a hora do acordar e hora de dormir até alcançar a hora desejada.

Nos adolescentes a medicação para as dificuldades de sono não está indicada, a não ser para tratamento de uma perturbação psiquiátrica de base ou em casos excepcionalmente graves. ${ }^{4}$ Mais uma vez o processo fundamental é trabalhar em conjunto com o adolescente e a família de forma a assegurar a higiene de sono, ${ }^{9,10,11}$ através de medidas gerais como (1) ir para a cama à mesma hora todas as noites, (2) levantar-se à mesma hora todos os dias, incluindo fins-de-semana, (3) evitar sestas, (4) limitar bebidas com cafeína ao jantar e após, (5) evitar exercício físico intenso 6 horas antes da hora de deitar, (6) evitar ler, ver televisão ou comer na cama, (7) se após cerca de 20 minutos de estar deitado não adormecer, levantar-se e dedicar-se a uma actividade tranquila, como ler, até se sentir novamente ensonado, deitando-se novamente e repetindo o esquema quantas vezes as necessárias.

\section{SONOLÊNCIA DIURNA}

A causa mais comum de sonolência diurna, muitas vezes erradamente interpretada por desinteresse ou desmotivação, é o sono insuficiente, incluindo alterações no padrão sono-vigília, ou simplesmente ausência de higiene de sono, ${ }^{2}$ com horários tardios de deitar e despertares cedo. Uma vez excluída esta situação e verificando-se que os horários de sono são adequados e suficientes, outras causas possíveis são as alterações qualitativas do sono nocturno, como ocorre, por exemplo, na apneia de sono. Nesta situação, a obstrução parcial ou completa das vias aéreas superiores conduz a uma redução de níveis de $\mathrm{O}_{2}$ e/ou aumento dos níveis de $\mathrm{CO}_{2}$, conduzindo a uma alteração na fisiologia do sono e a despertares intermitentes. O resultado é sonolência diurna com possível impacto no desenvolvimento cognitivo e comportamental da criança. Se houver suspeita de apneia de sono a situação deverá ser referenciada a consulta de otorrinolaringologia. Outras causas possíveis de interferir na qualidade de sono são doenças neurológicas, medicamentos, consumo de substâncias ou álcool. A terceira causa de sonolência diurna deve-se a um aumento das necessidades do sono, como na narcolepsia e síndrome de Kleine-Levin (SKL).

A narcolepsia tem uma prevalência de 1 em $2000^{2} \mathrm{e}$ predisposição familiar (1-2\% de risco de familiar em primeiro grau desenvolver esta perturbação). A situação inicia-se em regra na adolescência e tende a ser crónica. Na narcolepsia parece haver intrusão do sono 
REM no estado de vigília, resultando em episódios de sonolência diurna, alucinações hipnagógicas (como resultado do elemento sonho no sono REM), paralisia muscular (hipotonia súbita). Os episódios podem durar segundos a minutos e podem ocorrer também durante o sono, com alteração na sua arquitectura. A narcolepsia é difícil de diagnosticar em fases precoces pois os ataques de sonolência súbitos podem não estar ainda presentes. ${ }^{2} \mathrm{O}$ tratamento passa por rotinas de sono organizadas, incluindo sestas diurnas regulares e planeadas. Em termos de medicação, estimulantes como o metilfenidato mostraram ser úteis, tal como antidepressivos tricíclicos (por diminuírem o sono REM). ${ }^{4}$

Enquanto na narcolepsia a sonolência é persistente, no SKL, esta é intermitente. É uma síndrome $\operatorname{rara}^{5} \mathrm{e} \mathrm{ti}$ picamente começa após uma infecção ou experiência potencialmente geradora de stress em adolescentes do sexo masculino. Episódios acontecem de 2 a 3 vezes por ano, com duração de horas a semanas, caracterizados por hiperssónia, aumento do apetite, hiperssexualidade e estados sugestivos de ligeiro estado confusional agudo. A situação tende a reverter espontaneamente após alguns anos, existindo a possibilidade de medicação com estimulantes durante os episódios.

\section{PARASSÓNIAS}

As parassónias consistem num grupo de perturbações comuns durante a infância, caracterizadas por alterações no comportamento envolvendo o sono. ${ }^{9}$ Geralmente são fonte de grande preocupação para as famílias, pela sua exuberância, mas revestem-se de carácter benigno, sendo consideradas como parte integrante do desenvolvimento e tendendo a desaparecer com a idade. ${ }^{2}$ Neste artigo dividiremos as parassónias em perturbações relacionadas com (1) despertares parciais (terrores nocturnos, sonambulismo, sonilóquios), (2) pesadelos e (3) automatismos do sono.

As perturbações relacionadas com despertares parciais ocorrem quando há uma passagem súbita do sono NREM profundo para um sono NREM leve. No fundo são comportamentos relacionados com despertares que emergem da fase de sono de ondas lentas (ondas delta). Visto que a maior proporção deste sono ocorre no primeiro terço da noite, estes episódios acontecem nesta altura, em média entre 1 a 3 horas após o adormecer. Durante estes episódios as crianças parecem acordadas, mas não estão de facto despertas.

Os terrores nocturnos podem aparecer por volta dos 18 meses de vida, com pico entre os 4 e os 7 anos, ${ }^{9}$ sendo mais raros após estas idades. A criança senta-se repentinamente na cama, agitada, confusa, olhar fixo, por vezes com sintomas vegetativos acompanhantes, como palpitações, respiração irregular e diaforese. Parece inconsolável. O episódio dura segundos a minutos até a criança acalmar subitamente e adormecer novamente. De manhã não há recordação do episódio por parte da criança. A criança não deve ser acordada, devendo ser conduzida de novo para a cama após o episódio. A persistência de forma regular para além da idade pode estar associada por vezes a sintomas fóbicos e deve ser alvo de atenção especializada. ${ }^{12}$

O sonambulismo é relativamente comum, sendo que $30 \%$ das crianças em determinado momento experienciaram um episódio. ${ }^{4}$ É habitual em cerca de 2,5\% da população geral. ${ }^{2}$ Parece haver uma base familiar, relacionada com a proporção de sono de ondas lentas. ${ }^{2} \mathrm{Um}$ episódio pode durar até cerca de 10 minutos e as medidas mais importantes são providenciar um ambiente seguro para evitar acidentes (colisões com objectos, escadas, etc.).

Em relação ao tratamento o primeiro passo é tranquilizar a família acerca da natureza benigna dos episódios, com um prognóstico favorável com a maturação da criança. ${ }^{4}$ Medidas práticas envolvem a possibilidade de uma sesta à tarde de cerca de 30 a 60 minutos, diminuindo a necessidade de sono de ondas lentas durante a noite, reduzindo a frequência dos episódios. Deverá ser promovido um ambiente tranquilo e relaxante durante o adormecimento, bem como medidas de segurança referidas para episódios de sonambulismo.

Para episódios graves, frequentes, com implicação na qualidade do sono e consequentemente sonolência diurna, ou perigosos, com risco para a criança, deve ser ponderada a medicação, nomeadamente as benzodiazepinas ou os antidepressivos tricíclicos que diminuem o sono de ondas lentas. Os mais usados destes agentes são o diazepam, clonazepam e imipramina, ao deitar. ${ }^{4}$

Os pesadelos são eventos relacionados com o sono REM e que ocorrem normalmente na segunda metade da noite. São comuns entre os 3 e 6 anos de vida, afectando de 10 a $50 \%$ de crianças. ${ }^{2}$ Ao contrário dos terro- 
res nocturnos, uma criança quando acorda de um pesadelo está assustada, alerta, mas orientada e lucidamente consegue descrever o episódio, tendo dificuldade em adormecer novamente. Na manhã seguinte recorda-se do pesadelo. Se os pesadelos forem persistentes e houver dificuldade na tranquilização da criança pode ser necessário tratamento, que deverá ser orientado para a fonte das angústias da criança. O conteúdo do pesadelo pode fornecer informação sobre a situação geradora de angústia. A persistência de forma regular para lá da idade normal deverá ser alvo de atenção, bem como outros sintomas associados.

Os automatismos de sono caracterizam-se por movimentos regulares e rítmicos (como abanar da cabeça ou batimentos com a cabeça no colchão ou grades do berço). Ainda é bastante desconhecido o seu significado patológico, a generalidade dos estudos revela que se trata de episódios benignos que resolvem espontaneamente aos 3-4 anos de idade. ${ }^{2}$ A maioria dos autores descreve os automatismos como comportamentos de autoapaziguamento. ${ }^{2}$ Ocorrem sobretudo nas fases $1 \mathrm{e}$ 2 do sono NREM, e por vezes no sono REM, duram segundos e podem ocorrer diversas vezes durante a noite. $\mathrm{O}$ tratamento passa por medidas protectoras para evitar lesões.

Existem outras parassónias como as "alucinações» hipnagógicas e hipnopômpicas, que são pseudoalucinações ao deitar e ao acordar, respectivamente. São comuns e sem significado patológico. A enurese nocturna é considerada por alguns autores como uma parassónia, mas a sua discussão parece-nos deslocada do âmbito deste artigo.

\section{PROBLEMAS DO SONO ASSOCIADOS A OUTRA PERTURBAÇÃO PSIQUIÁTRICA OU MÉDICA}

Como já foi referido, é fundamental, perante um problema de sono, detectar se há outro tipo de patologia do foro psiquiátrico ou orgânico na base das queixas apresentadas. A regulação do sono e vigília parece envolver sistemas neuronais também responsáveis pelo controlo das emoções e comportamento. Não é de estranhar a elevada comorbilidade entre perturbações psiquiátricas e perturbações do sono, em crianças e adolescentes. Os problemas de sono são sintomas de alarme importantes para detectar uma patologia do foro emocional.
Uma das perturbações psiquiátricas que comummente cursa com alterações do sono é a Perturbação de Hiperactividade e Déficit de Atenção (PHDA), havendo, em muitas destas crianças referência a diminuição das necessidades de sono, dificuldades no adormecer, sono agitado e frequentes despertares nocturnos. Na depressão, os problemas de sono fazem parte dos critérios de diagnóstico para episódio depressivo major, quer por insónia (inicial, média ou terminal) ou, no caso de depressões atípicas, por hiperssónia. As dificuldades no sono são também frequentes nas perturbações de ansiedade, como perturbação de ansiedade generalizada, perturbação obsessivo-compulsiva ou perturbação de stress pós-traumático. Neste último caso os pesadelos recorrentes acerca da situação geradora de stress fazem, regra geral, parte do quadro clínico. Na perturbação bipolar existe uma diminuição franca das necessidades de sono, enquanto em perturbações psicóticas são frequentes queixas de ansiedade nocturna. Nas deficiências mentais ou perturbações globais de desenvolvimento, é frequente encontrarem-se alterações no padrão sono-vigília, relacionadas, provavelmente, com alterações neurobiológicas do SNC.

Existe ainda uma variedade de condições médicas e neurológicas que podem cursar com alterações do sono. O clínico, na sua avaliação, deve excluir a possibilidade destas situações estarem presentes. São exemplos destes casos a apneia do sono, asma, refluxo gastro-esofágico, obesidade, patologia tiroideia, doenças degenerativas, epilepsia, cefaleias, bem como a narcolepsia ou a SKL supramencionados.

\section{CONCLUSÕES}

Como revimos neste artigo, há uma grande variedade de problemas de sono no bebé, na criança e no adolescente, que podem ser encarados como um espectro de intensidade. Numa ponta do espectro temos dificuldades de adormecimento ou despertares nocturnos pontuais, que podem ser variações da normalidade (dependente também da percepção e atitude dos pais face a estas dificuldades) e na outra ponta problemas graves que podem interferir com o desenvolvimento normal.

Torna-se claro que é necessário ajudar os pais e fornecer orientações claras no sentido de promoção de padrões saudáveis de sono, bem como na detecção pre- 
coce de problemas de sono, de forma a que o sono possa ser encarado pelas crianças como uma experiência positiva.

Deverão ser encaminhadas para consulta de Pedopsiquiatria os casos em que as medidas psicopedagógicas não se apresentaram eficazes, bem como aqueles em que haja associação com outros sintomas psicopatológicos, ou em que pareça haver uma grande dificuldade dos pais em lidar com a situação, gerando uma situação insustentável do ponto de vista do equilíbrio familiar.

\section{REFERÊNCIAS BIBLIOGRÁFICAS:}

1. Davis KF, Parker KP, Montgomery GL. Sleep in infants and young children: Part one: normal sleep. J Pediatr Health Care 2004 Mar-Apr; 18 (2): 65-71.

2. Stein A, Barnes J in Rutter M, Taylor E. Child and Adolescent Psychiatry. 4th ed. Oxford: Blackwell; 2005.

3. Carvalho Bos S, Gomes A, Clemente V, Marques M, Pereira AT, Maia B, et al. Sleep and behavioral/emotional problems in children: a population-based study. Sleep Med 2009 Jan; 10 (1): 66-74.

4. Connor D, Meltzer B. Pediatric Psychopharmacology Fast Facts. New York: Norton \& Company; 2006.
5. Touchette E, Petit D, Tremblay RE, Montplaisir JY. Risk factors and consequences of early childhood dyssomnias: new perspectives. Sleep Med Rev 2009 Oct; 13 (5): 355-61.

6. Lewis M. Child and Adolescent Psychiatry: a comprehensive textbook. 3rd ed. Lippincott, Williams \& Wilkins Publishers; 2002.

7. Stahl S. Stahl's Essential Psychopharmacology. 3rd ed. Cambridge: Cambridge University Press; 2008.

8. Sadeh A, Sivan Y. Sleep problems during infancy. Eur J Pediatr 2009 Oct; 168 (10): 1159-64.

9. Davis KF, Parker KP, Montgomery GL. Sleep in infants and young children: Part two: common sleep problems. J Pediatr Health Care 2004 May-Jun; 18 (3): 130-7.

10. Healey D, France KG, Blampied NM. Treating sleep disturbance in infants: what generalizes? Behav Intervent 2009 Feb; 24 (1): 23-41.

11. Mindell JA, Meltzer LJ, Carskadon MA, Chervin RD. Developmental aspects of sleep hygiene: findings from the 2004 National Sleep Foundation Sleep in America Poll. Sleep Med 2009 Aug; 10 (7): 771-9.

12. Marcelli D. Infância e Psicopatologia. Lisboa: Climepsi; 2005.

Os autores declaram não possuir conflitos de interesse.

\section{ENDEREÇO PARA CORRESPONDÊNCIA}

Manuel Salavessa

E-mail: msalavessa@gmail.com

Paula Vilariça

E-mail: paula.vilarica@yahoo.com

\section{ABSTRACT}

\section{SLEEP PROBLEMS IN CHILDREN}

The importance and impact of sleep on health has earned more attention in the recent years, being sleep problems a common source of concern among parents. Sleep plays a key role not only biologically, but also emotionally and socially. Sleep problems can have a significant negative impact on children and their families. In this article we intend to deal with basic information to be used by clinicians when confronted with the parents' concerns about children's common sleep problems. We will focus on the fundamental aspects of normal sleep architecture and sleep patterns development, followed by an explanation about the clinically more relevant sleep problems in children and adolescents.

Keuwords: Sleep; Sleep Disorder; Child; Adolescent. 